\title{
Academician Alexander Mikhailovich Baldin (1926-2001)
}

Academician Alexander Mikhailovich Baldin, an outstanding scientist in the field of elementary particle physics and atomic nucleus, was born on February 26, 1926 in Moscow.

He graduated from locomotive technical secondary school and was enrolled in the Moscow Institute of Transport Engineers as a student. At that time, he had already realized that science is his true vocation. In 1946, he was invited, among other excellent students, to continue his education in a newly created Moscow Mechanics Institute of Ammunition, named later Moscow Engineering Physics Institute (MIFI).

After graduating from MIFI in 1949, he was assigned to the P.N. Lebedev Physical Institute of the USSR Academy of Sciences (FIAN), where he made his way from a junior research worker to a head of the theoretical division. He received the doctorate degree and became a full professor. He has matured as a scientist under the influence of a brilliant Pleiad of FIAN's physicists gathered by S.I. Vavilov. He considered D.V. Skobeltsyn and M.A. Markov to be his teachers. A.M. Baldin's first research work on the theory of particle motion in a cyclic accelerator had already then

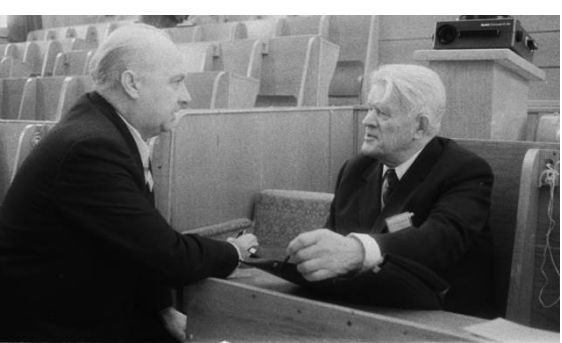

A.M. Baldin (left) and P.A. Cherenkov (right) attracted the attention of specialists and first of all of V.I. Veksler. These investigations, carried out jointly with V.V. Mikhailov, under M.S. Rabinovich were aimed at the solution of a wide range of problems related to the theory of cyclic accelerators and had underlain the physical substantiation of the Synchrophasotron which was the biggest accelerator of this type in the word at that time. They were completed with the creation of a method which had become classic and is being widely used till now in the calculations of accelerators.

It was just at that time that he was keen on mountaineering, where he had achieved brilliant results and had become a recognized authority. Mountains favored him to develop such distinguishing traits of his character as inflexibility, manliness and the capability of making rational decisions which were used in full measure in his scientific leadership and like so much in his life trials.

The start up of electron and proton accelerators in the late forties and during the fifties in the USSR required highly qualified physicists engaged in the theoretical elaboration and planning of experimental research programs who would profoundly understand the status and prospects of the development of both modern theory and experimental and accelerator engineering. A.M. Baldin had become one of the most brilliant and fruitfully working personality who fell under this category of Soviet physicists.

As long ago as in early fifties, due to the development of work at the FIAN electron synchrotron and on Markov's initiative, A.M. Baldin (partially in coopera-

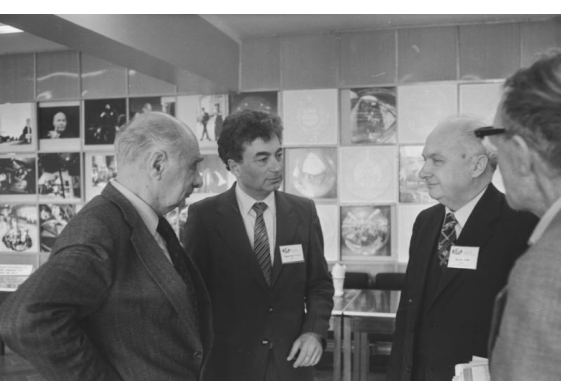

G.N. Flerov, Yu.Ts. Oganessian and A.M. Baldin (from left to right) tion with V.V. Mikhailov) carried out pioneering calculations of the cross sections of meson production on nucleons and nuclei when exposed to high-energy photons. A pole model taking into account anomalous magnetic moments of nucleons introduced into these calculations was subsequently substantiated and became an integral part of the dispersion re- 
lation method. The research work along these lines, awarded the State Prize in 1973, played an appreciable role in the development of strong interaction theory and the formation of the ideas about the existence of an intrinsic structure and excited states of nucleons, as well as in the development of the methods of an adequate description of processes involving strongly interacting particles. In just the same way as classic Hofstadter's experiments on electron scattering on nucleons and nuclei led to the idea about spatial extension of the hadron electric charge distribution, the study of the Compton scattering performed at FIAN and interpreted by Baldin in terms of the coefficients of nucleon electromagnetic polarizability showed that the elementary particles, which the nucleons are usually attributed to, are not only spatially extended systems, but also dynamically deformed ones. The discovery of this effect by authors' group was registered in 1957. Baldin's dispersion sum rule for the sum of the coefficients of nucleon electric and magnetic polarizability suggested in 1960 served as a basis for the first realistic estimate of the proton electric polarizability and is presently the major and most universal ingredient of all modern analyses of the data on the low energy Compton effect on nucleons. A mutual transformability of the known elementary particles, the possibility of their production and annihilation in the intermediate state of a reaction in question, is the fundamental feature of relativistic quantum dynamics that leads to a mutual dependence of the properties of different elementary particles.

An experiment on lepton decay of the phi meson accompanied by an intermediate transition of the massive particle to a photon was performed at JINR, on Baldin's initiative and with his participation. This discovery, registered in 1971, confirmed, in particular, the presence of a "hadronic" photon component which stipulates a hadron-like behavior of the cross sections for high-energy photon interactions with nucleons and nuclei, determining, in a figurative sense, "nuclear" properties of light.

By generalizing the notions of molecular optics, A.M. Baldin introduced into nuclear physics the notions

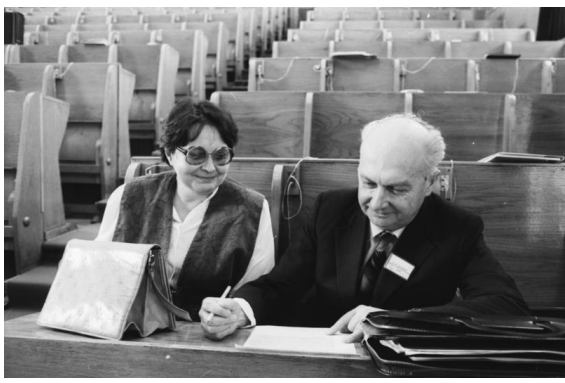

I.S. Baldina and A.M. Baldin of tensor and vector polarizability of nuclei which describe "optical anisotropy" of atomic nuclei with a non-zero spin. One of the most striking manifestations of this property was found to be a splitting of a giant dipole resonance of strongly deformed nuclei into two maxima corresponding to two types of collective dipole oscillations of nucleons: along and across the nucleus symmetry axis. A.M. Baldin obtained the first numerical estimates of the parameters of the optical anisotropy of atomic nuclei and predicted in such a way a number of effects to be observed. Afterwards this result was proved experimentally by American physicists who recognized Baldin's priority.

On M.A. Markov's initiative and with his active support, Alexander Mikhailovich was elected a Director of the Laboratory of High Energies of the Joint Institute for Nuclear Research in 1968. A young man, as he was at that time, became responsible for the formulation of a topical scientific program to be realized by the Laboratory's staff formed by V.I. Veksler, for the conservation and increase of the research facilities available and, first of all, for the "Veksler's heritage" - the Synchrophasotron. This unique machine, the creation of which cost infinite labor in severe post-war years, gave pioneering results on elementary particle physics. A whole generation of experimenters thus got a start to their creative life.

As a scientific leader, A.M. Baldin had to determine priorities of the development of the accelerator and experimental complexes of the Laboratory which required all his scientific talent, life experience, health and soul. Nuclear interactions at relativistic energies were chosen as a major research 
direction. To this end, under the guidance of A.M. Baldin, the Synchrophasotron was modified to a specialized accelerator of relativistic and polarized nuclei.

In the early 70s, A.M. Baldin determined long-term research goals in relativistic nuclear physics - a scientific trend of paramount importance for the national science - which arose at the meeting point of atomic nuclear physics and elementary particle physics. It was just aimed at setting up the application limits for the proton-neutron model of the atomic nucleus and the creation of the physical picture of nuclear matter at the level of sub-nucleonic constituents, i.e. quarks and gluons. For the first time in the international practice of accelerator engineering, beams of relativistic nuclei moving with velocities near the speed of light and of an energy of order of several $\mathrm{GeV}$ per nucleon were obtained at the Synchrophasotron in LHE.

The study of nucleus-nucleus collisions was ex-

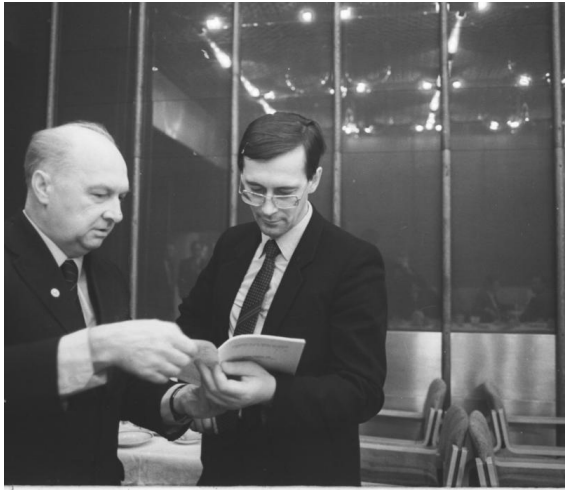

A.M. Baldin (left) and V.A. Matveev (right) tended to the energy range where the principles of the theory of relativity became predominant. A new research trend, relativistic nuclear physics, arose on the foundation of the achievements obtained in quantum field theory, elementary particle physics, nuclear physics and acceleration physics. The whole intellectual potential of Alexander Michailovich was essentially required to formulate and promote this new trend. Followed by Dubna examples, relativistic nuclear physics has become a prominent part of programs at the biggest accelerator centers of the USA, Europe, Russia and the JINR Member-States. A wide experimental program on particle physics is progressing in collaboration with the Institute of High Energy Physics (Protvino), the E. Fermi Laboratory (USA) and the European Organization for Nuclear Research (CERN).

In the period of the formation of relativistic nuclear physics, A. Baldin displayed his creative energy, scientific foresight, capacity for work. He was able to communicate with large groups of people, to persuade and unite them. The importance of these qualities could scarcely be exaggerated. Being a broadminded person of rare erudition in many facets of modern physics, deeply understanding modern physics experiments A.M. Baldin was highly respected by the scientific community all over the world. He did not only suggest ideas of new experiments, but he was also a di-

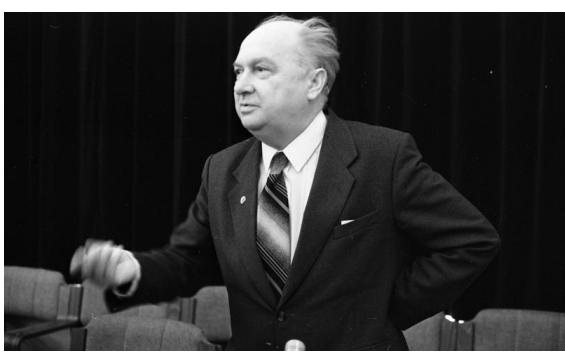

A.M. Baldin rect organizer and a tireless participant in them.

Baldin's prediction of the nuclear cumulative effect was the first success in this area of physics. The first experiments in Dubna showed that at an energy of several $\mathrm{GeV}$ the particle production in nuclear collisions is setting up in the asymptotic regime. It means the achievement of invariability of the physical picture of the secondary particle production in nuclear fragmentation with increasing collision energy or the achievement of the so-called limiting fragmentation of nuclei - the idea introduced by A.M. Baldin. The quark and gluon degrees of freedom begin to play an essential role in this area. A remarkable peculiarity of the nuclear fragmentation process is the extension of these properties also to particles produced behind the kinematic limit of free nucleon collisions, i.e. to cumulative particles. In the language of the parton model, this fact points out that nuclei contain multiquark states. 
The picture of the relativistic energy nuclear fragmentation is found to be in a perfect analogy with the hitherto known particular features of high energy proton-proton interactions and deep inelastic scattering of electrons on protons. The discovered empirical regularities enabled A.M. Baldin to introduce a universal distribution of partons in nuclei - the quark-parton structure function of the nucleus. This aroused considerable interest in the development of a theory for the description of a nucleus at distances shorter than the nucleon size (models of fluctons, short-range nucleon correlations, multiquark states in nuclei and so on).

A.M. Baldin suggested universal approaches to the description of not only spectra of single particles, but also the whole picture of an act of multiple particle production in nuclear collisions. He proposed to describe the nuclear interaction process in the four-velocity space starting from the principles of symmetry - self-similarity symmetry. The picture of multiple particle production is found to be analogous to a microscopic process of "point-like explosion". There was discovered and is being studied a universal law which makes it possible to describe cumulative processes, sub-threshold processes,

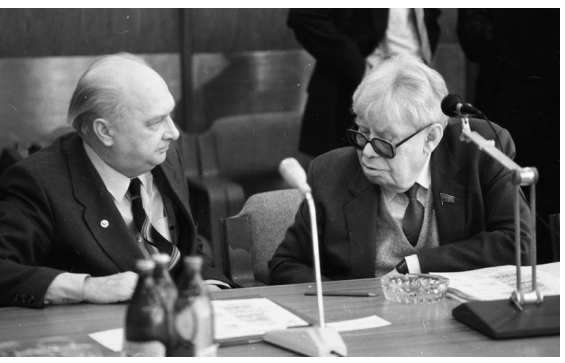

A.M. Baldin (left) and N.N. Bogoliubov (right) anti-nucleus production processes, as well as the ones that occur in the region of transition from nucleon to quark-gluon variables.

The results of this new trend - relativistic nuclear physics - together with the fundamental works of theorists of the school of N.N. Bogoliubov were united to clear up a dynamic role of the new quantum number "color" and the appropriate symmetry in pursuing the observed scale-invariant behavior of hadron and nuclear interactions with large momentum transfer. They were awarded the Lenin Prize in 1988.

Under the guidance of A.M. Baldin, the Laboratory accelerator complex developed successfully: there were constructed new ion sources and experimental sites of accelerated beams. Experiments with polarized deuteron beams formed a special trend of research; unique polarized neutron beams were obtained. Unique information about the spin structure of the deuteron - this "hydrogen atom of nuclear physics" - at inter-nucleon distances less than the nucleon radius was extracted from these experiments.

The results of the first period of investigations with relativistic nuclei enabled A.M. Baldin to suggest and justify the idea about constructing of a specialized accelerator - the Nuclotron - the magnetic system of which is based on the phenomenon of superconductivity. A.M. Baldin succeeded in uniting people around this task due to his talent for organization and his purposeful activity. Under his guidance, exceptionally complicated engineering problems were solved and first of all fast-cycling superconducting magnets and a helium liquefaction complex were constructed. The Nuclotron launching and development in the 90s opened up the way to qualitatively new possibilities of studying the properties of atomic nuclei. The science of the JINR Member-States, including Russian physics, acquired a powerful basis for first-class investigations on strong interaction physics.

A.M. Baldin paid the most serious attention to applications of the achievements of relativistic nuclear physics, Nuclotron's technologies, for the design of accelerator controlled nuclear-energetic apparatus, for transmutation of nuclear wastes as well as for cosmic flight radiation studies.

Academician A.M. Baldin's scientific and organizational activities were extremely versatile. He was the President of the Council on Electromagnetic Interactions and a member of the Bureau of the Nuclear Physics Department of the Russian Academy of Sciences, Editor-in-Chief of the journals "Physics of Elementary Particles and Atomic Nuclei" and "Physics of Particles and Nuclei Letters" as well as a member of the Editorial Boards of many scientific publications. Among conferences that 
were organized by Alexander Michailovich of special importance are the International Seminars on High-Energy Physics Problems started in 1969 with M.A. Markov's support. They were given an unofficial, somewhat witty, name "Baldin autumn".

A.M. Baldin devoted much time and effort to the training of scientific staff. Among his disciples are dozens of holders of the doctoral degree and candidate of sciences. He gave lectures at Moscow State University, Moscow Engineering Physics Institute, the JINR University Center, as well as at many international schools of physics. Baldin did a lot for preparing highly skilled specialists in JINR Member-States. In Dubna he founded his scientific school which was intended for theorists, experimenters, specialists of accelerator engineering.

In his talks and papers devoted to the general problems of the scientific research strategy, A.M. Baldin tirelessly emphasized the influence of the centers of fundamental science on scientific and technological progress and the potential of the country. His sense of high civic responsibility and deep understanding of the role of science in today's community impelled him, as a statesman, to recur to these problems.

The achievements of the outstanding scientist and a talented organizer of science were awarded with the Lenin Prize, the State Prize, the V.I. Veksler Prize of the Russian Academy of Sciences, as well as with govern-

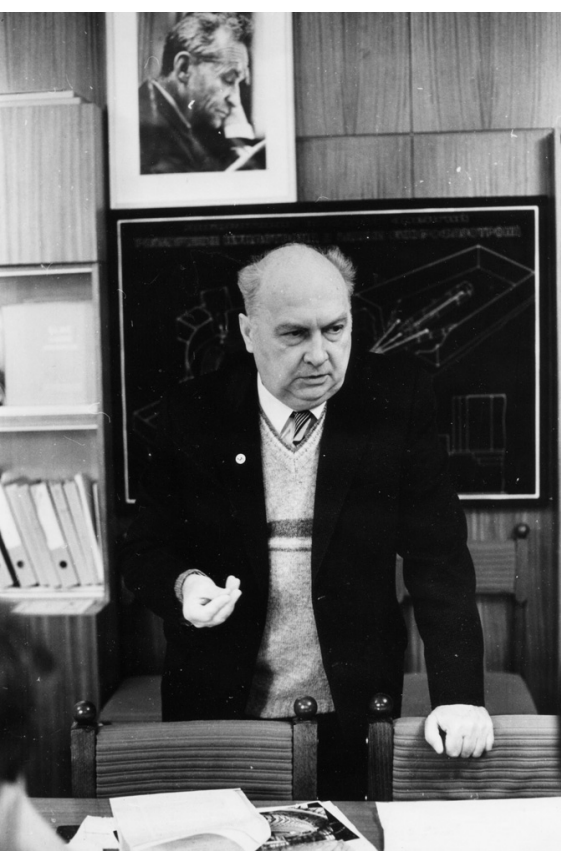

A.M. Baldin mental awards - the Order of the Red Banner of Labor, the Order of Merit, the Order of Service to the Fatherland, forth class. He was awarded orders and medals of the JINR Member States. A.M. Baldin was given the title of Honorary Citizen of the town of Dubna.

Alexander Michailovich gained fine reputation and sincere respect for his selfless service to the Science, high civic duty, true intelligence, as well as for his constant concern for people and social justice. He worked hard and effectively and he was very good for people.

Alexander Michailovich died on April 29, 2001 after serious illness.

\section{V.V. Burov}

\section{A.I. Malakhov}

September 2016, Dubna 